\title{
Late effects of pediatric hematopoietic stem cell transplantation on left ventricular function, aortic stiffness and myocardial tissue characteristics
}

Elisabeth H. M. Paiman ${ }^{1 *}$ (D), Marloes Louwerens², Dorine Bresters ${ }^{3,4}$, Jos J. M. Westenberg ${ }^{1}$, Qian Tao ${ }^{1}$, Rob J. van der Geest ${ }^{1}$, Arjan C. Lankester ${ }^{3}$, Arno A. W. Roest ${ }^{3+}$ and Hildo J. Lamb ${ }^{1+}$

\begin{abstract}
Background: Pediatric hematopoietic stem cell transplantation (HSCT) recipients are at increased risk of cardiovascular disease later in life. As HSCT survival has significantly improved, with a growing number of HSCT indications, tailored screening strategies for HSCT-related late effects are warranted. Little is known regarding the value of cardiovascular magnetic resonance (CMR) for early identification of high-risk patients after HSCT, before symptomatic cardiovascular disease manifests. This study aimed to assess CMR-derived left ventricular (LV) systolic and diastolic function, aortic stiffness and myocardial tissue characteristics in young adults who received HSCT during childhood.
\end{abstract}

Methods: Sixteen patients ( $22.1 \pm 1.5$ years) treated with HSCT during childhood and 16 healthy controls ( $22.1 \pm 1.8$ years) underwent 3 T CMR. LV systolic and diastolic function were measured as LV ejection fraction (LVEF), the ratio of transmitral early and late peak filling rate (E/A), the estimated LV filling pressure (E/Ea) and global longitudinal and circumferential systolic strain and diastolic strain rates, using balanced steady-state free precession cine CMR and $2 \mathrm{D}$ velocity-encoded CMR over the mitral valve. Aortic stiffness, myocardial fibrosis and steatosis were assessed with 2D velocity-encoded CMR, native T1 mapping and proton CMR spectroscopy ('H-CMRS), respectively.

Results: In the patient compared to the control group, E/Ea ( $9.92 \pm 3.42$ vs. $7.24 \pm 2.29, P=0.004)$ was higher, LVEF ( $54 \pm 6 \%$ vs. $58 \pm 5 \%, P=0.055)$ and global longitudinal strain (GLS) $(-20.7 \pm 3.5 \%$ vs. $-22.9 \pm 3.0 \%, P=0.063)$ tended to be lower, while aortic pulse wave velocity $(4.40 \pm 0.26 \mathrm{vs} .4 .29 \pm 0.29 \mathrm{~m} / \mathrm{s}, P=0.29)$, native $\mathrm{T} 1(1211 \pm 36 \mathrm{vs} .1227$ $\pm 28 \mathrm{~ms}, P=0.16)$ and myocardial triglyceride content $(0.47 \pm 0.18$ vs. $0.50 \pm 0.13 \%, P=0.202)$ were comparable. There were no differences between patients and controls in E/A ( $2.76 \pm 0.92 \mathrm{vs} .2 .97 \pm 0.91, P=0.60)$ and diastolic strain rates.

Conclusion: In young adults who received HSCT during childhood, LV diastolic function was decreased (higher estimated LV filling pressure) and LV systolic function (LVEF and GLS) tended to be reduced as compared to healthy controls, whereas no concomitant differences were found in aortic stiffness and myocardial tissue characteristics. When using CMR, assessment of LV diastolic function in particular is important for early detection of patients at risk of HSCT-related cardiovascular disease, which may warrant closer surveillance.

Keywords: Hematopoietic stem cell transplantation, Pediatric, Cardiovascular magnetic resonance, Systolic and diastolic function, Aortic stiffness, Diffuse fibrosis, Myocardial steatosis, T1 mapping

\footnotetext{
* Correspondence: E.H.M.Paiman@lumc.nl

${ }^{\dagger}$ Arno A. W. Roest and Hildo J. Lamb contributed equally to this work.

'Department of Radiology, Leiden University Medical Center, P.O. Box 9600,

postal zone C2-S, 2300 RC Leiden, The Netherlands

Full list of author information is available at the end of the article
}

(c) The Author(s). 2019 Open Access This article is distributed under the terms of the Creative Commons Attribution 4.0 International License (http://creativecommons.org/licenses/by/4.0/), which permits unrestricted use, distribution, and

reproduction in any medium, provided you give appropriate credit to the original author(s) and the source, provide a link to the Creative Commons license, and indicate if changes were made. The Creative Commons Public Domain Dedication waiver (http://creativecommons.org/publicdomain/zero/1.0/) applies to the data made available in this article, unless otherwise stated. 


\section{Background}

Hematopoietic stem cell transplantation (HSCT) recipients are exposed to several pre-transplant and/or HSCT-related therapies which may increase the risk of cardiovascular disease [1, 2]. As HSCT survival has significantly improved over the last decades $[3,4]$, with an increasing number of HSCT indications for both malignant and non-malignant disease [5], targeted follow-up strategies for the HSCT population are needed [6]. Recently, several international working groups have been established aimed at a greater understanding of the late effects including arterial disease and cardiac dysfunction $[7,8]$. Most of the available studies on late effects involve HSCT in adults. Adequate screening in young HSCT recipients is even more challenging [9].

According to current guidelines [10], pediatric HSCT recipients who have an increased susceptibility to complications later in life, based on pre-existing comorbidities, pre-transplant exposures, the HSCT preparative regimen, post-transplant complications such as graft-versus-host-disease, or relapse of the primary disease, are selected for patient-specific follow-up programs. Identification of imaging markers which indicate subclinical disease would be supportive in the detection of high-risk patient groups for closer monitoring or targeted therapy [6]. In general, clinical follow-up after cardiotoxic exposures comprises echocardiography for left ventricular (LV) systolic and diastolic function. Less is known regarding the value of cardiovascular magnetic resonance (CMR) for screening of late effects [11]. CMR may be suited for comprehensive evaluation of subclinical deteriorations within the cardiovascular system after HSCT, that may be present before overt LV functional abnormalities arise.

Pre-transplant or HSCT-related cardiotoxic exposures may cause endothelial damage leading to increased aortic stiffness and myocyte cell death with reactive interstitial fibrosis $[1,2]$. In addition, the immunosuppressive therapies in allogeneic HSCT increase the susceptibility to developing the metabolic syndrome at young age [12]. Aortic stiffening is known to occur in relation to normal, physiological ageing [13], but will be more progressive in response to hypertension, dyslipidemia or hyperglycemia [14] and possibly due to iron overload [15]. Increased aortic stiffness induces LV concentric remodeling and is recognized as an independent predictor for cardiovascular events [16]. Myocardial diffuse fibrosis in different types of cardiomyopathies is considered to reflect subclinical disease before cardiac dysfunction becomes manifest [17]. The metabolic derangements among allogeneic HSCT recipients may predispose to myocardial steatosis. In individuals with the metabolic syndrome, myocardial steatosis has been associated with LV remodeling [18].
We hypothesize that, when using CMR, subclinical deteriorations in LV function, aortic stiffness and/or myocardial tissue characteristics can be detected in young adults who received HSCT during childhood. Therefore, CMR may be suitable for early identification of patients at increased risk of developing HSCT-related cardiovascular disease. Accordingly, the aim of this study is to assess CMR-derived LV systolic and diastolic function, aortic stiffness and myocardial fibrosis and steatosis in young adults who have received pediatric HSCT and to compare these measures with those in healthy controls in the same age range.

\section{Methods \\ Study population}

The patient group consisted of young adults (18 to 25 years old), who received HSCT for malignant or non-malignant disease during childhood. Patients were recruited from the outpatient clinic for screening and treatment of late effects of childhood cancer and/or HSCT of the Internal Medicine Department (Leiden University Medical Centre, the Netherlands). Healthy controls were recruited by local advertising in Leiden University, the Netherlands. The control group was in the same age range as the patients and was sex-matched. Laboratory measures in the patient group were performed based on clinical indication and were typically measured within 1 year prior or after CMR examination. No blood samples were drawn in the healthy control group.

\section{CMR acquisition}

The study participants underwent $3 \mathrm{~T}$ CMR (Ingenia, Philips Healthcare, Best, the Netherlands), with a dStream Torso anterior coil and a FlexCoverage posterior coil in the table top, resulting in up to 32 coil elements for signal reception. The protocol consisted of standard electrocardiographic (ECG)-triggered two-, three- and four-chamber and short-axis cine balanced steady-state free precession (bSSFP) CMR and ECG-gated gradient-echo 2D velocity-encoded CMR over the mitral valve to quantify LV structure, systolic and diastolic function; 2D velocity-encoded CMR transecting the aortic arch and abdominal aorta to derive aortic stiffness; cardiac native T1 mapping to assess diffuse fibrosis and proton cardiovascular magnetic resonance spectroscopy ( $\left.{ }^{1} \mathrm{H}-\mathrm{CMRS}\right)$ to measure the myocardial triglyceride content. For standardization of the measurement of myocardial triglyceride content, all participants were asked to fast for $6 \mathrm{~h}$ and the CMR examinations were scheduled at fixed times (evenings). No CMR contrast material was used.

For the bSSFP cines, typical field-of-view (FOV) was $350 \times 350 \mathrm{~mm}^{2}$ (long-axis) and $400 \times 352 \mathrm{~mm}^{2}$ (short-axis), acquired voxel size $2.0 \times 1.6 \mathrm{~mm}^{2}$ (long-axis) and $1.5 \times 1.5 \mathrm{~mm}^{2}$ (short-axis), slice thickness $8 \mathrm{~mm}$, echo/ repetition time (TE/TR) $1.5 / 3.0 \mathrm{~ms}$, flip angle $45^{\circ}$, number of phases 30 (long-axis) and 35 (short-axis). For 
short-axis bSSFP cine, the complete LV was imaged, using 14-16 slices, depending on the size of the LV, with 2 slices per breath-hold. Free-breathing through-plane 2D velocity-encoded (venc $100 \mathrm{~cm} / \mathrm{s}$ ) CMR was acquired perpendicular to the mitral valve, with FOV $350 \times 350 \mathrm{~mm}^{2}$, acquired voxel size $2.5 \times 2.5 \mathrm{~mm}^{2}$, slice thickness $8 \mathrm{~mm}$, flip angle $10^{\circ}$, TE/TR $2.9 / 4.6 \mathrm{~ms}$, number of phases 40 .

Aortic pulse wave velocity (PWV) was quantified as a measure of aortic stiffness. For aortic PWV, first, a double-oblique sagittal scout view of the aorta was obtained. Then, two free-breathing through-plane 2D velocity-encoded CMR scans were acquired, one transecting the ascending aorta (venc $150 \mathrm{~cm} / \mathrm{s}$ ) and one transecting the abdominal aorta, above the aortic bifurcation (VENC $100 \mathrm{~cm} / \mathrm{s}$ ). Typical imaging parameters were: FOV $350 \times 282 \mathrm{~mm}^{2}$, slice thickness $8 \mathrm{~mm}$, acquired voxel $2.8 \times 2.8 \mathrm{~mm}^{2}$, flip angle $20^{\circ}$, TE/TR $2.5 / 4.4$ $\mathrm{ms}$, temporal resolution $10 \mathrm{~ms}$.

Native T1 mapping was acquired in the mid-ventricular short-axis slice, with breath-holding, using the $5 \mathrm{~s}(3 \mathrm{~s}) 3 \mathrm{~s}$ modified Look-Locker inversion recovery (MOLLI) scheme. Typical imaging parameters were: FOV $350 \times 300 \mathrm{~mm}^{2}$, slice thickness $8 \mathrm{~mm}$, acquired voxel size $2.1 \times 2.1 \mathrm{~mm}^{2}$, flip angle $20^{\circ}$, TE/TR 1.1/2.3 ms, SENSitivity Encoding (SENSE) factor 2. ${ }^{1} \mathrm{H}$-MRS was performed as described previously [19]. In summary, a voxel of $40 \times 15 \times 25 \mathrm{~mm}^{3}$ was placed in the interventricular septum. For the acquisition with and without water suppression, 48 and 6 signal averages were obtained, respectively. ECG-triggering was used to acquire ${ }^{1} \mathrm{H}$-CMRS at 200 $\mathrm{ms}$ after the R-wave, and a respiratory navigator, tracking the lung-liver interface, for acquisition at end-expiration. A high permittivity pad was placed on the chest for improved signal-to-noise ratio.

\section{Image analysis}

CMR data were analyzed using MASS Research Software V2016-EXP (Leiden University Medical Center, the Netherlands) for LV structure and function and aortic PWV, custom-made software for further analysis of the aortic velocity-time curves [20], Medis Suite 3.0 (Medis Medical Imaging systems, Leiden, the Netherlands) for LV systolic and diastolic strain (QStrain 2.0) and native T1 (QMap 2.2.18), and the Java-based magnetic resonance user interface (jMRUI v5.0; MRUI Consortium) for ${ }^{1} \mathrm{H}$-CMRS. The image analysis was blinded to patient or healthy control status.

For LV mass and volumes, the endocardial and epicardial LV borders were manually outlined in the end-diastolic and end-systolic phase, with exclusion of the LV papillary muscles. For the feature tracking based strain calculations, the manually annotated endocardial LV borders were automatically tracked throughout the cardiac cycle. Global longitudinal strain (GLS), global longitudinal peak systolic strain rate (GLSR-S) and global longitudinal early peak diastolic strain rate (GLSR-E) were calculated based on the two-, three- and four-chamber cine images. Global circumferential strain (GCS), global circumferential peak systolic strain rate (GCSR-S) and global circumferential early peak diastolic strain rate (GCSR-E) were extracted from the mid-ventricular short-axis cine slice (Fig. 1). The ratio of the transmitral early and late peak filling rate (E/A ratio) and the transmitral early peak maximum velocity were derived from the 2D velocity-encoded scans (Fig. 1), as described previously [21]. The transmitral filling rate was measured after correction for the through-plane background velocity of the LV myocardial wall. Furthermore, the early diastolic mitral septal tissue velocity (Ea) was extracted from the four-chamber cine images. Subsequently, the estimated LV filling pressure (ratio of transmitral early peak maximum velocity without through-plane myocardial motion correction and early diastolic mitral septal tissue velocity) was calculated (E/Ea ratio).

Aortic PWV was calculated by dividing the distance between ascending and abdominal aorta by the transit time of the onset of the systolic velocity wave front (Fig. 2), as described previously [20]. In short, the aortic path length was measured manually along the aortic centerline on the double-oblique sagittal aorta scout scan. The onset of the systolic wave front was automatically determined from the resulting velocity graph by the intersection point of the constant horizontal diastolic velocity and upslope of the systolic wave front, modeled by linear regression (using the velocity values between 20 and $80 \%$ of the total range) along the upslope. T1 maps were constructed after manual in-plane motion correction of the T1 images, using a pixel-wise, mono-exponential three-parameter fit for the T1 relaxation curve. For the measurement of native T1, a region-of-interest in the mid-ventricular septum was drawn (Fig. 2). Myocardial triglyceride content was expressed as the percentage of triglyceride methyl (at $0.9 \mathrm{ppm}$ ) and triglyceride methylene (at $1.3 \mathrm{ppm}$ ) relative to the sum of the triglyceride signal and the unsuppressed water signal (at $4.7 \mathrm{ppm}$ ) (Fig. 2) [22].

\section{Statistics}

Statistical analyses were performed in SPSS 23 (International Business Machines, Armonk, New York, USA). Normal distribution was checked using the Shapiro-Wilk test. Differences between groups were tested for statistical significance using the Student's t-test or the Mann-Whitney $\mathrm{U}$ test for normally and non-normally distributed variables, respectively. Patients with prior HSCT vs. controls were compared and additional analyses were performed for patients with HSCT for malignancies vs. controls. Levels for statistical significance were set at $P<0.05$ and all tests were 


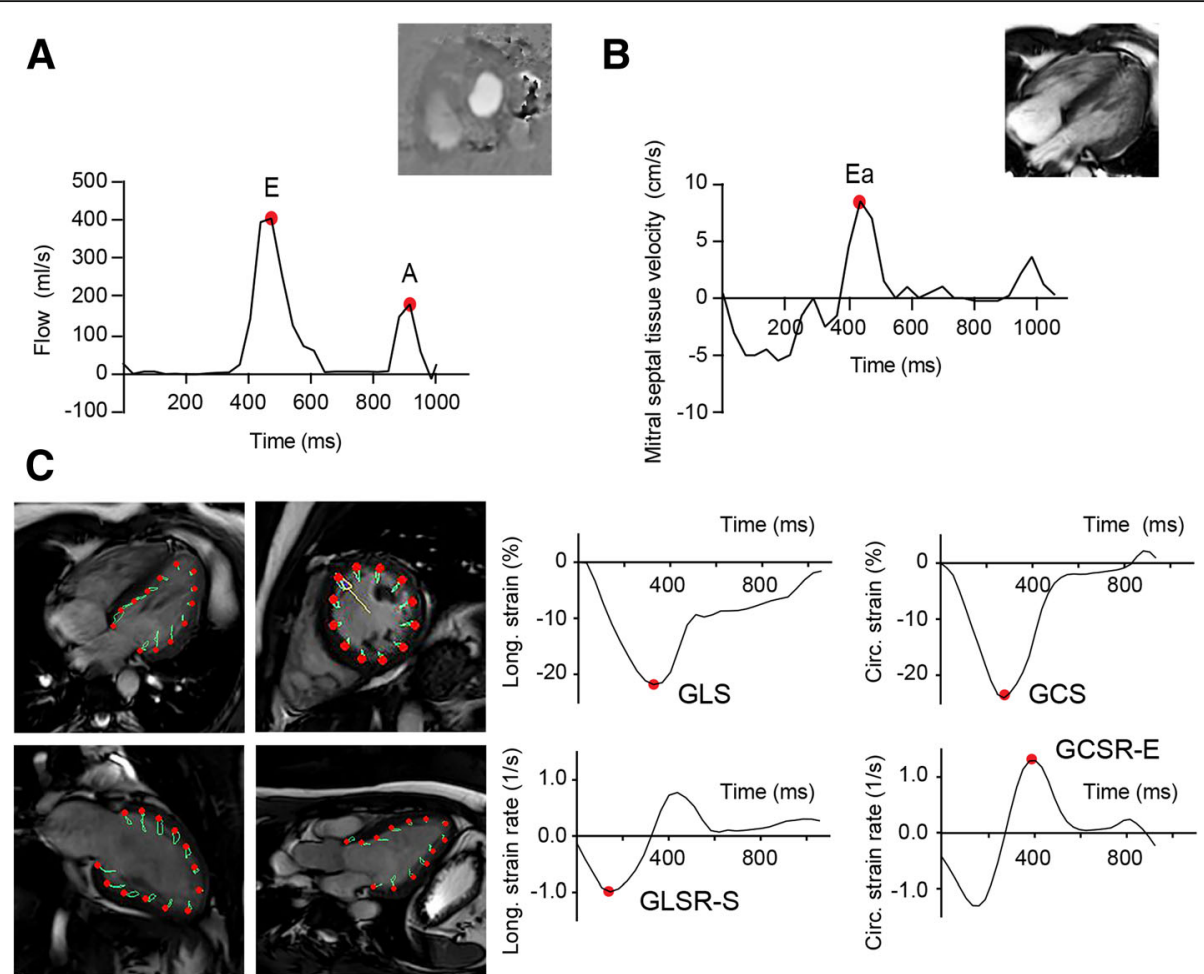

Fig. 1 A 22-year-old man, who was transplanted for a non-malignant bone marrow failure disorder at the age of 8 years. a the transmitral E/A ratio (early peak filling rate/late peak filling rate) was measured using 2D velocity-encoded CMR (left panel) and Ea (early peak diastolic mitral septal tissue velocity) was derived from the four-chamber long-axis relaxation (right panel). LV filling pressure was estimated by the ratio of the transmitral early peak maximum velocity and the early peak diastolic mitral septal tissue velocity. b From two, three- and four-chamber and midventricular short-axis cine CMR (left panel), the longitudinal and circumferential strain and strain rate curves were extracted (right panel). The myocardial features at the endocardial borders (red dots), which were automatically tracked throughout the cardiac cycle (green lines), were manually annotated in the end-diastolic and end-systolic phase. GLS: global longitudinal strain; GCS: global circumferential strain; GLSR-S: global longitudinal peak systolic strain rate; GCSR-E: global circumferential early peak diastolic strain rate

two-sided. Additionally, we performed a post-hoc power analysis (alfa 0.05 , two-sided).

\section{Results}

In total, sixteen patients $(22.1 \pm 1.5$ years, $11 / 16$ (69\%) men) and sixteen healthy controls (22.1 \pm 1.8 years, 11 / 16 (69\%) men) were included and analyzed. In one patient, ${ }^{1} \mathrm{H}$-CMRS data were not analyzed due to insufficient quality. Also, in one patient $2 \mathrm{D}$ velocity-encoded CMR over the mitral valve was not acquired due to imaging time constraints. Height and body surface area (BSA) in the patient group were lower compared to the healthy control group. The anthropometric characteristics are presented in Table 1.

In ten patients, HSCT was indicated for a malignant disorder. Seven patients were treated with anthracyclines prior to HSCT, eight received total body irradiation (typically unfractionated 7.5 Gy or $2 \times 6$ Gy) and fourteen were given high-dose cyclophosphamide $\left(>1 \mathrm{~g} / \mathrm{m}^{2}\right)$ for HSCT conditioning. Five patients had a ferritin level above $250 \mu \mathrm{mol} / \mathrm{L}$. In two of them, cardiac $\mathrm{T} 2^{*}$ was assessed upon clinical indication. In both, the $\mathrm{T} 2 \%$ values were not suggestive of myocardial iron deposition. Clinical and biochemical patients characteristics are presented in Tables 2 and 3.

Patients as compared to the healthy controls had a higher E/Ea ratio $(9.92 \pm 3.42$ vs. $7.24 \pm 2.29, P=0.004)$, while the E/A ratio $(2.76 \pm 0.92$ vs. $2.97 \pm 0.91, P=0.599)$ and diastolic strain rates were comparable. There was a trend towards a lower LV ejection fraction (LVEF) (54 \pm 6 vs. $58 \pm 5 \%, P=0.055)$ and lower GLS $(-20.7 \pm 3.5$ vs. $-22.9 \pm 3.0 \%, P=0.063)$, whereas GCS was preserved $(-23.2 \pm 3.6$ vs. $-23.9 \pm 3.5 \%, P=0.587)$. Stroke volume ( 83 \pm 15 vs. $101 \pm 17 \mathrm{~mL}, P=0.003$ ) but also cardiac output $(5.2 \pm 1.0$ vs. $6.5 \pm 1.0 \mathrm{~L}, P=0.001)$ and cardiac index $\left(2.9 \pm 0.6\right.$ vs. $\left.3.4 \pm 0.5 \mathrm{~L} / \mathrm{m}^{2}, P=0.021\right)$ were lower. In contrast, aortic PWV $(4.40 \pm 0.26$ vs. $4.29 \pm 0.29 \mathrm{~m} / \mathrm{s}, P$ $=0.288), \mathrm{LV}$ concentricity $(0.62 \pm 0.10$ vs. $0.61 \pm 0.08 \mathrm{~g} / \mathrm{mL}$, $P=0.867)$, native T1 (1211 \pm 36 vs. $1227 \pm 28 \mathrm{~ms}, P=0.158)$ and myocardial triglyceride content $(0.47 \pm 0.18$ vs. $0.50 \pm 0.13 \%, P=0.202$ ) were comparable. CMR results are presented in Table 4 and Fig. 3 .

When comparing the subgroup with HSCT for malignancies with controls, the higher $\mathrm{E} / \mathrm{Ea}$ persisted 
A
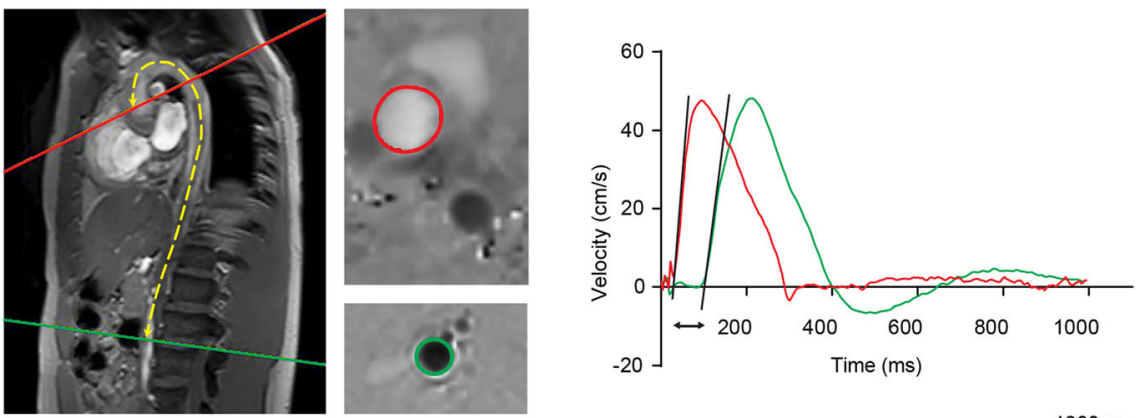

B
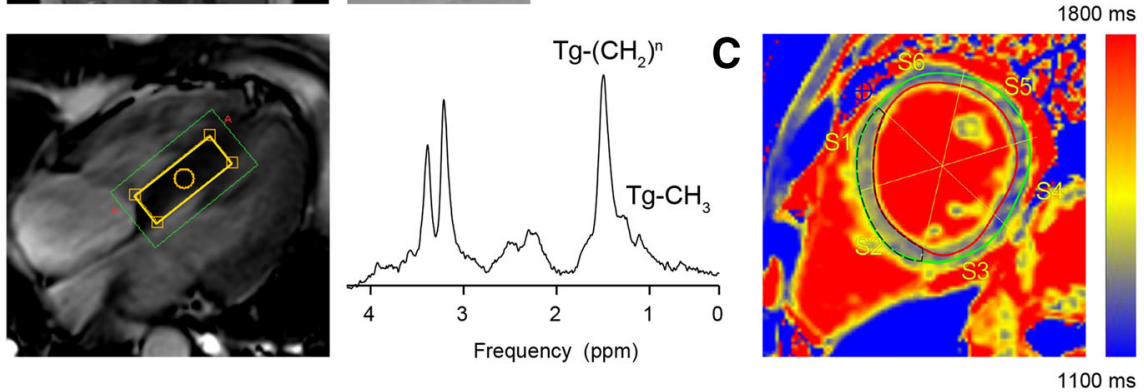

Fig. 2 The same patient as in Fig. 1 is presented. a Aortic pulse wave velocity was calculated from through-plane 2D velocity-encoded CMR transecting the ascending aorta (red) and the abdominal aorta, above the aortic bifurcation (green) (left panel), according to: aortic pulse wave velocity $=\Delta x / \Delta t$, with $\Delta x$ : the distance between the ascending and abdominal aorta (yellow dotted line) and $\Delta t$ : transit time of the onset of the systolic velocity wave front (black arrow) (right panel). b Proton-cardioavascular magnetic resonance spectroscopy ( ${ }^{1} \mathrm{H}$-CMRS) was used to measure the myocardial triglyceride content. The voxel of interest was placed in the mid-ventricular septum (yellow box) (left panel). Myocardial triglyceride content was calculated as $\mathrm{Tg}-\left(\mathrm{CH}_{2}\right)^{\mathrm{n}}$ and $\mathrm{Tg}-\mathrm{CH}_{3}$ relative to the sum of the triglyceride and the unsuppressed water signal (not shown). Triglycerides were measured using the water-suppressed spectrum (right panel). c Native T1 was measured in the mid-ventricular septal segments in short-axis view (black, dotted region of interest)

$(10.33 \pm 3.55$ vs. $7.24 \pm 2.29, P=0.013)$. Additionally, LVEF $(52 \pm 4$ vs. $58 \pm 5 \%, P=0.008)$, GLS $(-19.8 \pm 3.3$ vs. $-22.9 \pm 3.0 \%, P=0.022)$ and GCSR-S $(-1.13 \pm 0.15$ vs. $-1.32 \pm 0.261 / \mathrm{s}, P=0.047)$ were lower. For the HSCT recipients treated for malignancies, aortic PWV was: $4.39 \pm 0.18 \mathrm{~m} / \mathrm{s} \quad(P=0.36$ vs. controls $), \mathrm{LV}$ concentricity: $0.63 \pm 0.10 \mathrm{~g} / \mathrm{mL}(P=0.73)$, native T1: $1208 \pm 36 \mathrm{~ms}(P=0.138)$ and myocardial triglyceride content: $0.53 \pm 0.21(P=0.69)$.

\section{Post-hoc power analysis}

We had 0.90 power to detect a statistically significant difference if the HSCT population would have had an aortic PWV of $4.64 \mathrm{~m} / \mathrm{s}$ or higher $(+0.33 \mathrm{~m} / \mathrm{s}$ higher as compared to the healthy controls), myocardial triglyceride content of $0.69 \%$ or higher $(+0.19 \%)$ and native $\mathrm{T} 1$ of $1268 \mathrm{~ms}$ or higher $(+39 \mathrm{~ms})$. These differences in aortic PWV, myocardial triglyceride content and native T1 are comparable to approximately 3, 20 and 35 years of normal aging, respectively [23-25].

\section{Discussion}

Our study showed that young adults with a history of pediatric HSCT, as compared to healthy controls in the same age range, have subclinical impairments in LV diastolic function and tend to have a lower LV systolic function, whereas aortic stiffness and myocardial tissue characteristics are comparable. Our results indicate that CMR-derived LV diastolic parameters, rather than aortic PWV, cardiac native T1 or myocardial triglyceride

Table 1 Demographic and anthropometric characteristics

\begin{tabular}{llll}
\hline & Pediatric HSCT recipients $(n=16)$ & Healthy controls $(n=16)$ & $P$ value \\
\hline Men, $n(\%)$ & $11(69 \%)$ & $11(69 \%)$ & 1.000 \\
Age, $y$ & $22.1 \pm 1.5$ & $22.1 \pm 1.8$ & 1.000 \\
Length, $\mathrm{cm}$ & $173 \pm 7$ & $179 \pm 8$ & $0.017^{*}$ \\
Weight, $\mathrm{kg}$ & $66.6 \pm 10.9$ & $72.1 \pm 6.9$ & 0.095 \\
Body mass index (BMI), $\mathrm{kg} / \mathrm{m}^{2}$ & $22.4 \pm 3.6$ & $22.5 \pm 2.7$ & 0.929 \\
Body surface area (BSA), $\mathrm{m}^{2}$ & $1.78 \pm 0.16$ & $1.89 \pm 0.11$ & $0.029^{*}$ \\
\hline
\end{tabular}

Values are presented as numbers (percentages) or means \pm standard deviations. ${ }^{*} P<0.05$. BSA based on the Mosteller formula 
Table 2 Clinical patient characteristics

\begin{tabular}{|c|c|}
\hline & Pediatric HSCT recipients $(n=16)$ \\
\hline \multicolumn{2}{|l|}{ Transplant-related characteristics } \\
\hline Age at time of $\mathrm{HSCT}$, years & $7.2 \pm 5.3$ \\
\hline Time after HSCT, years & $14.8 \pm 5.0$ \\
\hline Malignant disorder, $n$ (\%) & $10(62.5 \%)$ \\
\hline ALL or AML & 8 \\
\hline MDS or CML & 2 \\
\hline Non-malignant disorder, $n$ (\%) & $6(37.5 \%)$ \\
\hline Hematological disease & 4 \\
\hline Other & 2 \\
\hline Anthracycline therapy, $n$ (\%) & $7(43.8 \%)$ \\
\hline$<300 \mathrm{mg} / \mathrm{m}^{2}$ & 5 \\
\hline$\geq 300 \mathrm{mg} / \mathrm{m}^{2}$ & 2 \\
\hline Radiotherapy, n (\%) & $8(50.0 \%)$ \\
\hline Total body irradiation & 7 \\
\hline Chest irradiation & 1 \\
\hline Cyclophosphamide therapy, n (\%) & $14(87.6 \%)$ \\
\hline$<1 \mathrm{~g} / \mathrm{m}^{2}$ & 1 \\
\hline$\geq 1 \mathrm{~g} / \mathrm{m}^{2}$ & 13 \\
\hline Allogeneic HSCT, $n$ (\%) & $15(94 \%)$ \\
\hline HLA-identical sibling & 6 \\
\hline Other related or unrelated donor & 9 \\
\hline Graft versus host disease, $n(\%)$ & $5(31.3 \%)$ \\
\hline Acute & 2 \\
\hline Chronic & 3 \\
\hline \multicolumn{2}{|l|}{ Clinical parameters } \\
\hline Systolic blood pressure, $\mathrm{mmHg}$ & $121 \pm 12$ \\
\hline Diastolic blood pressure, $\mathrm{mmHg}$ & $76 \pm 9$ \\
\hline Pulse, beats per minute & $75 \pm 12$ \\
\hline
\end{tabular}

Values are presented as numbers (percentages) or means \pm standard deviations. HSCT hematopoietic stem cell transplantation, ALL acute lymphatic leukemia, $A M L$ acute myeloid leukemia, MDS myelodysplastic syndrome, $C M L$ chronic myeloid leukemia

content, may be early markers of cardiovascular deterioration after HSCT.

\section{LV function}

The estimated LV filling pressure was found to be increased after childhood HSCT, which is indicative of reduced LV diastolic function. When LV myocardial relaxation progressively deteriorates, LV filling pressure becomes increased to compensate for the impaired LV diastolic filling [26]. Accordingly, in the patients who received HSCT during childhood, the E/A ratio was comparable as in the controls, whereas LV filling pressure was higher. In contrast to the impairments in LV diastolic function, the decrease in LV systolic function in the HSCT group compared to the control group was non-significant. This observation is in keeping with prior longitudinal studies using echocardiography, which showed that diastolic dysfunction precedes systolic dysfunction in response to cardiotoxic exposures [27, 28]. Anthracycline-related cardiomyopathy is considered to result from free radical formation, the consequent mitochondrial dysfunction and myofibrillar disarray, and eventual myocyte necrosis; in turn, with the ongoing remodeling of the injured heart, functional impairments may progressively develop [2]. Furthermore, irradiation may cause microcirculatory damage, leading to myocardial ischemia and cell death [2]. However, despite the reduced LV diastolic function, there was no interstitial fibrosis based on native T1 mapping. Furthermore, aortic stiffening or myocardial steatosis did not appear contributing factors to the abnormalities in LV diastolic function. Hence, when using CMR, LV diastolic parameters in particular may be important in the monitoring of HSCT-related cardiovascular deteriorations.

In our study, the trend for lower LVEF and significantly lower cardiac index in the pediatric HSCT recipients compared to the controls seemed to be related to impairments in GLS rather than GCS. GLS and GCS, respectively, can be interpreted as measures of the contractility of the cardiomyocytes in oblique orientation in the subendocardium and those in circumferential arrangement in the mesomyocardium $[29,30]$. Accordingly, our results may suggest that the HSCT-related therapies predominately affect the subendocardial myocardium, and not the midwall. In the subgroup with a history of a malignant disorder, longitudinal and circumferential systolic strain-derived parameters were lower as compared to healthy controls; this is consistent with previous studies which assessed LV systolic strain parameters in patients receiving anthracyclines [31, 32].

We hypothesized that aortic stiffening or altered myocardial tissue characteristics would be early markers of cardiovascular disturbances after HSCT, preceding manifest LV dysfunction. Of interest, as chemotherapy is considered to disrupt mitochondrial function and irradiation has been demonstrated to induce microcirculatory damage [2], several other CMR-derived parameters may have potential for the early detection of cardiac effects of HSCT, for example phosphorus CMRS ( $\left.{ }^{31} \mathrm{P}-\mathrm{CMRS}\right)$ for the assessment of myocardial energetics and perfusion imaging [33].

\section{Aortic stiffness}

No differences were observed in aortic stiffness between the HSCT study population and the healthy controls. Based on previous studies, we expected to find a significantly higher aortic stiffness, at least in the patients with HSCT for a malignant disorder, because of the pre-transplant cardiotoxic exposures. Anthracycline and/or chest irradiation 
Table 3 Biochemical patient characteristics

\begin{tabular}{|c|c|c|}
\hline & Pediatric HSCT recipients $(n=16)$ & Normal values \\
\hline Fasting glucose, $\mathrm{mmol} / \mathrm{L}$ & $5.1 \pm 0.8$ & $3.1-6.4$ \\
\hline Total cholesterol, $\mathrm{mmol} / \mathrm{L}$ & $4.90 \pm 1.20$ & $3.90-7.30$ \\
\hline HDL-cholesterol, mmol/L & $1.30 \pm 0.32$ & $0.80-2.30$ \\
\hline LDL-cholesterol, mmol/L & $2.69 \pm 0.87$ & $0.00-3.37$ \\
\hline Triglycerides, mmol/L & $1.87 \pm 1.29$ & $0.80-2.30$ \\
\hline \multirow[t]{2}{*}{ Alanine aminotransferase (ALT), U/L } & Men: $32 \pm 17$ & $0-45$ \\
\hline & Women: $15 \pm 4$ & $0-34$ \\
\hline \multirow[t]{2}{*}{ Aspartate aminotransferase (AST), U/L } & Men: $31 \pm 16$ & $0-35$ \\
\hline & Women: $21 \pm 2$ & $0-31$ \\
\hline \multirow[t]{2}{*}{ Gamma-glutamyl transpeptidase (GGT), U/L } & Men: $40 \pm 24$ & $0-55$ \\
\hline & Women: $19 \pm 10$ & $0-38$ \\
\hline Free thyroxin (FT4), pmol/L & $15.9 \pm 2.6$ & $12.0-22.0$ \\
\hline Thyroid-stimulating hormone (TSH), mU/L & $2.695 \pm 1.472$ & $0.300-4.800$ \\
\hline eGFR (CKD-EPI) > 60 mL/min/1.73m², n (\%) & $16(100 \%)$ & NA \\
\hline \multirow[t]{2}{*}{ Ferritin, $\mu \mathrm{mol} / \mathrm{L}$} & Men: 187, $183(61,910)$ & $35-260$ \\
\hline & Women: 31, $437(8,792)$ & $10-150$ \\
\hline Ferritin > $250 \mu \mathrm{mol} / \mathrm{L}, n(\%)$ & $5(31.3 \%)$ & NA \\
\hline \multirow[t]{2}{*}{ Hemoglobin, mmol/L } & Men: $9.0 \pm 0.7$ & $8.5-11.0$ \\
\hline & Women: $8.4 \pm 0.9$ & $7.5-10.0$ \\
\hline
\end{tabular}

Values are presented as numbers (percentages), mean \pm standard deviation or median, interquartile range (minimum, maximum), if the distribution was skewed. Separate values are reported for men $(n=11)$ and women $(n=5)$, if applicable. HSCT hematopoietic stem cell transplantation

in the treatment of the primary cancer may cause endothelial damage due to the generation of reactive oxygen species $[34,35]$. Also, the conditioning in allogeneic HSCT, including total body irradiation and/or chemotherapy, is considered to initiate disruption of the endothelium [36]. Subsequently, the structural changes in the vascular matrix and the disturbed endothelial function may increase the vascular tone of the arterial wall [2]. In addition, metabolic disturbances caused by the immune dysregulation and iron overload are well recognized adverse effects of HSCT [12, 37]. There is extensive evidence for an elevated aortic stiffness due to hypertension, dyslipidemia and hyperglycemia in the general population [14]. The role of iron overload in endothelial dysfunction is less evident, although iron chelation in coronary artery disease has been shown to improve endothelium-dependent vasodilation [38].

Previous longitudinal studies in patients with breast cancer, lymphoma or leukemia showed that aortic PWV increases by approximately 1.5 to 2-fold upon anthracyclines in the first 4 to 6 months, also for low or moderate dosages, compared to the pre-treatment measurements [39, 40]. Another study measured a higher carotid artery stiffness in children who had received allogeneic HSCT, for malignant or non-malignant disease, even though this study population showed only minor, subclinical metabolic derangements, and no other cardiovascular impairments were detected [41]. However, the increased aortic stiffness due to cardiotoxic exposures may decrease after the cardiotoxic therapy has been discontinued, as was shown in breast cancer patients who were followed before and 1, 4 and 14 months after anthracycline or trastuzumab chemotherapy [42]. We measured aortic PWV after a mean time of $14.8 \pm$ 5.0 years after HSCT. Possibly, there may have been changes in aortic PWV acutely after pediatric HSCT, which may have normalized after several years.

\section{Myocardial tissue characteristics}

There were no differences in myocardial tissue characteristics and LV concentricity between the HSCT study population and the healthy controls, which otherwise would have been indicative of LV remodeling. To our knowledge, this is the first study in which native T1, as a measure of interstitial fibrosis, and myocardial triglyceride content, as a measure of metabolic remodeling, have been assessed in young adults with prior HSCT. It should be noted that diffuse fibrosis can be estimated based on calculating native T1 or the extracellular volume fraction (ECV); the latter requires CMR contrast administration [17]. Recent CMR studies have demonstrated that both native T1 and ECV are increased in middle-aged patients with prior anthracycline therapy $[43,44]$. Other studies in adolescents exposed to anthracyclines, however, reported native $\mathrm{T} 1$ and/or ECV values within the normal range $[45,46]$; nonetheless, a 
Table 4 CMR parameters

\begin{tabular}{|c|c|c|c|}
\hline & Pediatric HSCT recipients $(n=16)$ & Healthy controls $(n=16)$ & $P$ value \\
\hline \multicolumn{4}{|l|}{ LV mass and dimensions } \\
\hline Mass, $g$ & $95 \pm 18$ & $108 \pm 23$ & 0.078 \\
\hline Mass/BSA, $g / m^{2}$ & $53 \pm 9$ & $57 \pm 10$ & 0.328 \\
\hline End-diastolic volume, $\mathrm{mL}$ & $155 \pm 29$ & $176 \pm 30$ & 0.052 \\
\hline End-diastolic volume/BSA, mL/m² & $87 \pm 14$ & $93 \pm 13$ & 0.238 \\
\hline Mass/end-diastolic volume, $\mathrm{g} / \mathrm{mL}$ & $0.62 \pm 0.10$ & $0.61 \pm 0.08$ & 0.867 \\
\hline \multicolumn{4}{|l|}{ LV systolic function } \\
\hline Stroke volume, $\mathrm{mL}$ & $83 \pm 15$ & $101 \pm 17$ & $0.003^{*}$ \\
\hline Cardiac output, L & $5.2 \pm 1.0$ & $6.5 \pm 1.0$ & $0.001^{*}$ \\
\hline Cardiac index, $\mathrm{L} / \mathrm{m}^{2}$ & $2.9 \pm 0.6$ & $3.4 \pm 0.5$ & $0.021^{*}$ \\
\hline Ejection fraction, \% & $54 \pm 6$ & $58 \pm 5$ & 0.055 \\
\hline Global longitudinal strain, \% & $-20.7 \pm 3.5$ & $-22.9 \pm 3.0$ & 0.063 \\
\hline Global circumferential strain, $\%$ & $-23.2 \pm 3.6$ & $-23.9 \pm 3.5$ & 0.587 \\
\hline Longitudinal peak systolic strain rate, $1 / \mathrm{s}$ & $-0.95 \pm 0.20$ & $-1.06 \pm 0.21$ & 0.137 \\
\hline Circumferential peak systolic strain rate, $1 / \mathrm{s}$ & $-1.21 \pm 0.22$ & $-1.32 \pm 0.36$ & 0.216 \\
\hline \multicolumn{4}{|l|}{ LV diastolic function } \\
\hline E/A ratio & $2.76 \pm 0.92$ & $2.97 \pm 0.91$ & $0.599 \neq$ \\
\hline Estimated LV filling pressure & $9.92 \pm 3.42$ & $7.24 \pm 2.29$ & $0.004 \neq$ \\
\hline Longitudinal early diastolic strain rate, $1 / \mathrm{s}$ & $1.01 \pm 0.26$ & $1.15 \pm 0.35$ & 0.224 \\
\hline Circumferential early diastolic strain rate, $1 / \mathrm{s}$ & $1.28 \pm 0.29$ & $1.38 \pm 0.35$ & 0.396 \\
\hline \multicolumn{4}{|l|}{ LV myocardial tissue characteristics } \\
\hline Myocardial triglyceride content, \% & $0.47 \pm 0.18$ & $0.50 \pm 0.13$ & $0.202 \neq$ \\
\hline Native $\mathrm{T} 1$ relaxation time, $\mathrm{ms}$ & $1211 \pm 36$ & $1227 \pm 28$ & 0.158 \\
\hline \multicolumn{4}{|l|}{ Aortic stiffness } \\
\hline Aortic pulse wave velocity, $\mathrm{m} / \mathrm{s}$ & $4.40 \pm 0.26$ & $4.29 \pm 0.29$ & 0.288 \\
\hline
\end{tabular}

correlation was found between the cumulative anthracycline dose and native T1 and ECV [46]. In contrast to these observations suggestive of diffuse fibrosis, focal fibrosis, visualized with late gadolinium enhancement CMR, is uncommon after anthracycline exposure, even when systolic function is subnormal [44-47]. In our HSCT study population as compared to the controls, we did not find a higher native T1, even though a lower LVEF was measured. Also, when analyzing the patients who received HSCT for a malignant indication separately, no difference with the control group was found. Therefore, in contrast to middle-aged patients with prior anthracycline therapy as described in other studies, we may conclude that there is no myocardial fibrosis, at least not substantial, in young adults with a history of childhood HSCT as compared to healthy controls.

From previous studies it is known that the metabolic syndrome, presumably due to changes in myocardial fatty acid uptake and/or oxidation, is associated with myocardial steatosis [18, 48]. Although allografting increases the risk of early development of the metabolic syndrome [12], our HSCT study population aged 18-25 years had on average no obesity, impaired fasting glucose or hypertriglyceridemia. Possibly, myocardial steatosis may arise after HSCT at middle-age, when metabolic disorders may have developed, while myocardial triglyceride content remains within the normal ranges at younger age. Based on our findings, we may conclude that myocardial triglyceride content does not represent an early marker of cardiometabolic disease after pediatric HSCT in the young adult population.

\section{Strengths}

Strength of this study is the comprehensive CMR evaluation, including measures of both LV systolic and diastolic function, vascular function and myocardial tissue characteristics. CMR rather than ultrasound is the most 

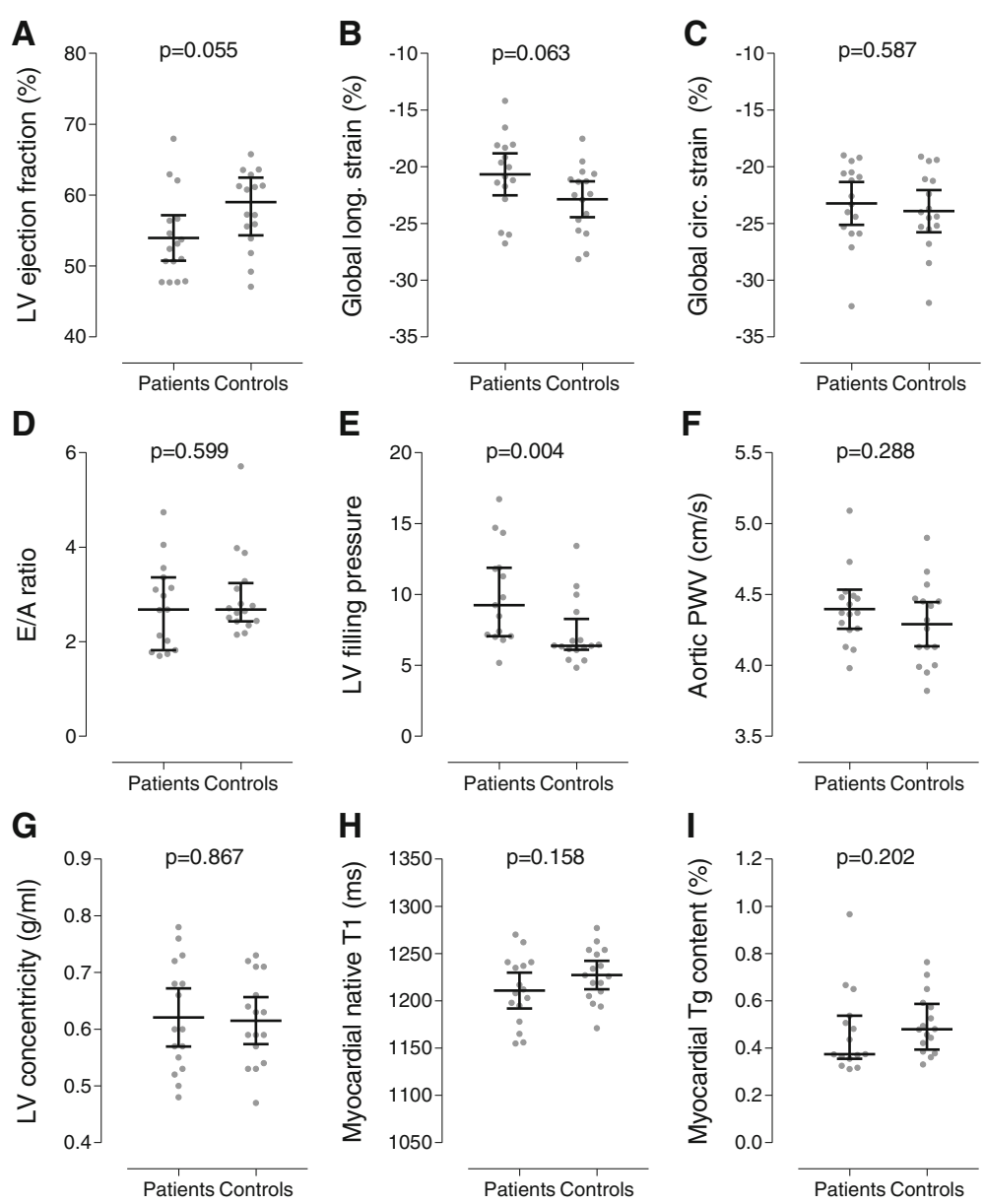

Fig. 3 CMR measurements with means and 95\% confidence intervals and median and interquartile ranges for normally and non-normally distributed data, respectively. Despite a non-significantly lower left ventricular (LV) systolic function (a-c) and lower LV diastolic function (d-e) as indicated by the increased estimated LV filling pressure, the CMR parameters for aortic stiffness ( $\mathbf{f}$ ) and LV structure $(\mathbf{g})$ and myocardial tissue characteristics (h-i) were comparable for the patients who received HSCT and the healthy controls. Abbreviations as in Table 4

accurate non-invasive modality to quantify aortic stiffness [49]. Diffuse fibrosis was measured using native T1 mapping, which is a non-contrast technique; contrast-enhanced CMR can be considered less suitable for screening programs. Cardiac ${ }^{1} \mathrm{H}$-CMRS is the gold standard for non-invasive assessment of myocardial steatosis [48]. In clinical practice, echocardiography is commonly used for follow-up after cardiotoxic therapies [11]. Compared to echocardiography, little is known regarding the value of CMR for cardiovascular screening after HSCT.

\section{Limitations}

Our study did not comprise CMR before and shortly after HSCT, but had a cross-sectional design because of ethical considerations. Therefore, we cannot exclude temporary deteriorations in, for example, aortic stiffness. Our study population was too small to evaluate the patient group with HSCT for non-malignant disease separately. The observed abnormalities in LV diastolic function and the non-significantly lower LV systolic function seemed to be driven by the patients who had received HSCT for a malignant disease. However, based on our study we cannot rule out late LV functional impairment after HSCT for non-malignant disease. Also, we were not able to assess the correlations between the different HSCT conditioning regimens, graft-versus-host-disease or iron overload and cardiovascular measures. However, this study was aimed at identifying CMR parameters which may show deteriorations before overt cardiovascular disease develops, and not to identify HSCT-related factors which may be helpful in late cardiovascular disease risk stratification. Echocardiography was not applied in the present study, which would have allowed for comparison of CMR with echocardiography for the early detection of cardiac effects of HSCT.

As a measure of interstitial fibrosis, ECV may be preferable. The native $\mathrm{T} 1$ value may be affected by several 
processes other than edema or increased myocardial collagen in the extracellular compartment [17]. Hence, based on the native T1 measurements, we may not be able to exclude the presence of diffuse fibrosis in post-HSCT patients. However, as all patients were clinically monitored for cardiac iron deposition and as myocardial steatosis was ruled out based on the ${ }^{1} \mathrm{H}$-MRS measurements, it is unlikely that fibrosis related increases in native $\mathrm{T} 1$ might have been cancelled out by myocardial iron or fat related native $\mathrm{T} 1$ decreases.

\section{Conclusion}

In young adults who received HSCT during childhood, LV diastolic function was decreased (higher estimated LV filling pressure) and LV systolic function (LVEF and GLS) tended to be reduced as compared to healthy controls, whereas no concomitant differences were found in aortic stiffness (aortic PWV) and myocardial tissue characteristics (native T1 and myocardial triglyceride content). Therefore, when using CMR, the assessment of LV diastolic function in particular is important for early detection of patients at risk of HSCT-related cardiovascular disease. Further research, including longitudinal CMR measurements, is needed to show the predictive value of subclinical LV functional abnormalities for the development of symptomatic cardiovascular disease after HSCT. Also, comparative studies of imaging modalities should reveal whether CMR-derived LV function has additive value for screening programs when performed next to the current echocardiography-based follow-up of the cardiovascular late effects of HSCT. For example, CMR may be used for the selection of patients who require frequent follow-up by standard echocardiography.

\footnotetext{
Abbreviations

${ }^{1} \mathrm{H}$-CMRS: Proton cardiovascular magnetic resonance spectroscopy; BMI: Body mass index; BSA: Body surface area; bSSFP: Balanced steady-state free precession; CMR: Cardiovascular magnetic resonance; E/A ratio: Ratio of the transmitral early and late peak filling rate; E/Ea: Estimated left ventricular filling pressure; Ea: Early diastolic mitral septal tissue velocity; ECG: Electrocardiogram; ECV: Extracellular volume; EF: Ejection fraction; FOV: Field-of-view; GCS: Global circumferential strain; GCSR-E: Global circumferential early peak diastolic strain rate; GCSR-S: Global circumferential peak systolic strain rate; GLS: Global longitudinal strain; GLSR-E: Global longitudinal early peak diastolic strain rate; GLSR-S: Global longitudinal peak systolic strain rate; HSCT: Hematopoietic stem cell transplantation; LV: Left ventricle/left ventricular; LVEF: Left ventricular ejection fraction; MOLLI: Modified Look-Locker inversion recovery; PWV: Pulse wave velocity; SENSE: SENSitivity Encoding; TE: Echo time; TR: Repetition time; VENC: Velocity-encoding
}

\section{Acknowledgements}

Not applicable.

\section{Funding}

Not applicable.

\section{Availability of data and materials}

The datasets generated during and/or analyzed during the current study are available from the corresponding author on reasonable request.

\section{Authors' contributions}

EHMP drafted the manuscript. AAWR, HJL and ML were major contributors for writing the manuscript. AAWR and ACL designed the study. JJMW and HJL developed the scanning protocol. ML, DB and EHMP were responsible for patient and volunteer recruitment. EHMP acquired the data. EHMP, RJvdG, QT, JJMW and HJL were involved in the data analysis. All authors critically reviewed and approved the manuscript.

\section{Ethics approval and consent to participate}

The study was approved by the local institutional review board (Leiden University Medical Center, the Netherlands) and complies with the Declaration of Helsinki. All participants provided written informed consent prior to enrolment into the study.

\section{Consent for publication \\ Not applicable.}

\section{Competing interests}

The authors declare that they have no competing interests.

\section{Publisher's Note}

Springer Nature remains neutral with regard to jurisdictional claims in published maps and institutional affiliations.

\section{Author details}

${ }^{1}$ Department of Radiology, Leiden University Medical Center, P.O. Box 9600, postal zone C2-S, 2300 RC Leiden, The Netherlands. ${ }^{2}$ Department of Internal Medicine, Leiden University Medical Center, P.O. Box 9600, postal zone C7-Q, 2300 RC Leiden, The Netherlands. ${ }^{3}$ Department of Pediatrics, Leiden

University Medical Center, Leiden, The Netherlands. ${ }^{4}$ Princess Máxima Center for Pediatric Oncology, Utrecht, The Netherlands.

Received: 28 May 2018 Accepted: 4 December 2018

Published online: 17 January 2019

\section{References}

1. Uderzo C, Pillon M, Corti P, Tridello G, Tana F, Zintl F, et al. Impact of cumulative anthracycline dose, preparative regimen and chronic graftversus-host disease on pulmonary and cardiac function in children 5 years after allogeneic hematopoietic stem cell transplantation: a prospective evaluation on behalf of the EBMT pediatric diseases and late effects working parties. Bone Marrow Transplant. 2007;39(11):667-75.

2. Lipshultz SE, Adams MJ, Colan SD, Constine LS, Herman EH, Hsu DT, et al. Long-term cardiovascular toxicity in children, adolescents, and young adults who receive cancer therapy: pathophysiology, course, monitoring, management, prevention, and research directions: a scientific statement from the American Heart Association. Circulation. 2013;128(17):1927-95.

3. Pulte D, Gondos A, Brenner H. Trends in 5- and 10-year survival after diagnosis with childhood hematologic malignancies in the United States, 1990-2004. J Natl Cancer Inst. 2008;100(18):1301-9.

4. Chima RS, Daniels RC, Kim MO, Li D, Wheeler DS, Davies SM, et al. Improved outcomes for stem cell transplant recipients requiring pediatric intensive care. Pediatr Crit Care Med. 2012;13(6):e336-42.

5. Niederwieser D, Baldomero H, Szer J, Gratwohl M, Aljurf M, Atsuta Y, et al. Hematopoietic stem cell transplantation activity worldwide in 2012 and a SWOT analysis of the worldwide network for blood and marrow transplantation group including the global survey. Bone Marrow Transplant. 2016;51(6):778-85

6. Battiwalla M, Tichelli A, Majhail NS. Long-term survivorship after hematopoietic cell transplantation: roadmap for research and care. Biol Blood Marrow Transplant. 2017;23(2):184-92.

7. Battiwalla M, Hashmi S, Majhail N, Pavletic S, Savani BN, Shelburne N. National Institutes of Health hematopoietic cell transplantation late effects initiative: developing recommendations to improve survivorship and longterm outcomes. Biol Blood Marrow Transplant. 2017;23(1):6-9.

8. Armenian SH, Chemaitilly W, Chen M, Chow EJ, Duncan CN, Jones LW, et al. National Institutes of Health hematopoietic cell transplantation late effects initiative: the cardiovascular disease and associated risk factors working group report. Biol Blood Marrow Transplant. 2017;23(2):201-10.

9. Pulsipher MA, Skinner R, McDonald GB, Hingorani S, Armenian SH, Cooke KR, et al. National Cancer Institute, National Heart, lung and blood institute/ 
pediatric blood and marrow transplantation consortium first international consensus conference on late effects after pediatric hematopoietic cell transplantation: the need for pediatric-specific long-term follow-up guidelines. Biol Blood Marrow Transplant. 2012;18(3):334-47.

10. Chow EJ, Anderson L, Baker KS, Bhatia S, Guilcher GM, Huang JT, et al. Late effects surveillance recommendations among survivors of childhood hematopoietic cell transplantation: a Children's oncology group report. Biol Blood Marrow Transplant. 2016;22(5):782-95.

11. Shankar SM, Marina N, Hudson MM, Hodgson DC, Adams MJ, Landier W, et al. Monitoring for cardiovascular disease in survivors of childhood cancer: report from the cardiovascular disease task force of the Children's oncology group. Pediatrics. 2008;121(2):e387-96.

12. Taskinen M, Saarinen-Pihkala UM, Hovi L, Lipsanen-Nyman M. Impaired glucose tolerance and dyslipidaemia as late effects after bone-marrow transplantation in childhood. Lancet. 2000;356(9234):993-7.

13. McEniery CM, Yasmin HIR, Qasem A, Wilkinson IB, Cockcroft JR, et al. Normal vascular aging: differential effects on wave reflection and aortic pulse wave velocity: the Anglo-Cardiff Collaborative Trial (ACCT). J Am Coll Cardiol. 2005:46(9):1753-60.

14. Terentes-Printzios D, Vlachopoulos C, Xaplanteris P, loakeimidis N, Aznaouridis K, Baou K, et al. Cardiovascular risk factors accelerate progression of vascular aging in the general population: results from the CRAVE study (cardiovascular risk factors affecting vascular age). Hypertension. 2017;70(5):1057-64

15. Cheung YF, Chan GC, Ha SY. Arterial stiffness and endothelial function in patients with beta-thalassemia major. Circulation. 2002;106(20):2561-6.

16. Ben-Shlomo Y, Spears M, Boustred C, May M, Anderson SG, Benjamin EJ, et al. Aortic pulse wave velocity improves cardiovascular event prediction: an individual participant meta-analysis of prospective observational data from 17,635 subjects. J Am Coll Cardiol. 2014;63(7):636-46.

17. Puntmann VO, Peker E, Chandrashekhar Y, Nagel E. T1 mapping in characterizing myocardial disease: a comprehensive review. Circ Res. 2016; 119(2):277-99.

18. Rayner JJ, Banerjee R, Holloway CJ, Lewis AJM, Peterzan MA, Francis JM, et al. The relative contribution of metabolic and structural abnormalities to diastolic dysfunction in obesity. Int J Obes. 2018;42(3):441-7.

19. de Heer P, Bizino MB, Versluis MJ, Webb AG, Lamb HJ. Improved cardiac proton magnetic resonance spectroscopy at $3 \mathrm{~T}$ using high permittivity pads. Investig Radiol. 2016;51(2):134-8.

20. Grotenhuis HB, Westenberg JJ, Steendijk P, van der Geest RJ, Ottenkamp J, Bax JJ, et al. Validation and reproducibility of aortic pulse wave velocity as assessed with velocity-encoded MRI. J Magn Reson Imaging. 2009;30(3): 521-6.

21. Brandts A, Bertini M, van Dijk EJ, Delgado V, Marsan NA, van der Geest RJ, et al. Left ventricular diastolic function assessment from three-dimensional three-directional velocity-encoded MRI with retrospective valve tracking. J Magn Reson Imaging. 2011;33(2):312-9.

22. de Heer P, Bizino MB, Lamb HJ, Webb AG. Parameter optimization for reproducible cardiac (1) H-MR spectroscopy at 3 tesla. J Magn Reson Imaging. 2016;44(5):1151-8.

23. Ohyama Y, Teixido-Tura G, Ambale-Venkatesh B, Noda C, Chugh AR, Liu CY, et al. Ten-year longitudinal change in aortic stiffness assessed by cardiac MRI in the second half of the human lifespan: the multi-ethnic study of atherosclerosis. Eur Heart J Cardiovasc Imaging. 2016;17(9):1044-53.

24. van der Meer RW, Rijzewijk LJ, Diamant M, Hammer S, Schar M, Bax JJ, et al. The ageing male heart: myocardial triglyceride content as independent predictor of diastolic function. Eur Heart J. 2008;29(12):1516-22.

25. Roy C, Slimani A, de Meester C, Amzulescu M, Pasquet A, Vancraeynest D, et al. Age and sex corrected normal reference values of T1, T2 T2* and ECV in healthy subjects at 3T CMR. J Cardiovasc Magn Reson. 2017;19(1):72.

26. Zile MR, Baicu CF, Gaasch WH. Diastolic heart failure--abnormalities in active relaxation and passive stiffness of the left ventricle. N Engl J Med. 2004; 350(19):1953-9.

27. Tassan-Mangina S, Codorean D, Metivier M, Costa B, Himberlin C, Jouannaud $C$, et al. Tissue Doppler imaging and conventional echocardiography after anthracycline treatment in adults: early and late alterations of left ventricular function during a prospective study. Eur J Echocardiogr. 2006;7(2):141-6.

28. Nagy AC, Cserep Z, Tolnay E, Nagykalnai T, Forster T. Early diagnosis of chemotherapy-induced cardiomyopathy: a prospective tissue Doppler imaging study. Pathol Oncol Res. 2008;14(1):69-77.
29. Ingels NB Jr. Myocardial fiber architecture and left ventricular function. Technol Health Care. 1997:5(1-2):45-52.

30. Dorri F, Niederer PF, Lunkenheimer PP, Anderson RH. The architecture of the left ventricular myocytes relative to left ventricular systolic function. Eur J Cardiothorac Surg. 2010;37(2):384-92.

31. Ong G, Brezden-Masley C, Dhir V, Deva DP, Chan KKW, Chow CM, et al. Myocardial strain imaging by cardiac magnetic resonance for detection of subclinical myocardial dysfunction in breast cancer patients receiving trastuzumab and chemotherapy. Int J Cardiol. 2018.

32. Lunning MA, Kutty S, Rome ET, Li L, Padiyath A, Loberiza F, et al. Cardiac magnetic resonance imaging for the assessment of the myocardium after doxorubicin-based chemotherapy. Am J Clin Oncol. 2015;38(4):377-81.

33. Hudsmith LE, Neubauer $S$. Magnetic resonance spectroscopy in myocardial disease. JACC Cardiovasc Imaging. 2009;2(1):87-96.

34. Keltai K, Cervenak L, Mako V, Doleschall Z, Zsary A, Karadi I. Doxorubicin selectively suppresses mRNA expression and production of endothelin-1 in endothelial cells. Vasc Pharmacol. 2010;53(5-6):209-14.

35. Soucy KG, Attarzadeh DO, Ramachandran R, Soucy PA, Romer LH, Shoukas $A A$, et al. Single exposure to radiation produces early anti-angiogenic effects in mouse aorta. Radiat Environ Biophys. 2010;49(3):397-404.

36. Woywodt A, Scheer J, Hambach L, Buchholz S, Ganser A, Haller H, et al. Circulating endothelial cells as a marker of endothelial damage in allogeneic hematopoietic stem cell transplantation. Blood. 2004;103(9):3603-5.

37. de Witte $T$. The role of iron in patients after bone marrow transplantation. Blood Rev. 2008;22(Suppl 2):S22-8.

38. Duffy SJ, Biegelsen ES, Holbrook M, Russell JD, Gokce N, Keaney JF Jr, et al. Iron chelation improves endothelial function in patients with coronary artery disease. Circulation. 2001;103(23):2799-804.

39. Chaosuwannakit N, D'Agostino R Jr, Hamilton CA, Lane KS, Ntim WO, Lawrence J, et al. Aortic stiffness increases upon receipt of anthracycline chemotherapy. J Clin Oncol. 2010;28(1):166-72.

40. Drafts BC, Twomley KM, D'Agostino R Jr, Lawrence J, Avis N, Ellis LR, et al. Low to moderate dose anthracycline-based chemotherapy is associated with early noninvasive imaging evidence of subclinical cardiovascular disease. JACC Cardiovasc Imaging. 2013;6(8):877-85.

41. Turanlahti MI, Taskinen M, Saarinen-Pihkala U, Jokinen EV. Time-related arterial changes after allogeneic hematopoietic stem cell transplantation in children. Pediatr Res. 2013;73(6):777-82.

42. Grover S, Lou PW, Bradbrook C, Cheong K, Kotasek D, Leong DP, et al. Early and late changes in markers of aortic stiffness with breast cancer therapy. Intern Med J. 2015;45(2):140-7.

43. Jordan JH, Vasu S, Morgan TM, D'Agostino RB Jr, Melendez GC, Hamilton CA, et al. Anthracycline-associated T1 mapping characteristics are elevated independent of the presence of cardiovascular comorbidities in Cancer survivors. Circ Cardiovasc Imaging. 2016;9(8).

44. Neilan TG, Coelho-Filho OR, Shah RV, Feng JH, Pena-Herrera D, Mandry D, et al. Myocardial extracellular volume by cardiac magnetic resonance imaging in patients treated with anthracycline-based chemotherapy. Am J Cardiol. 2013;111(5):717-22.

45. Toro-Salazar OH, Gillan E, O'Loughlin MT, Burke GS, Ferranti J, Stainsby J, et al. Occult cardiotoxicity in childhood cancer survivors exposed to anthracycline therapy. Circ Cardiovasc Imaging. 2013;6(6):873-80.

46. Tham EB, Haykowsky MJ, Chow K, Spavor M, Kaneko S, Khoo NS, et al, Diffuse myocardial fibrosis by T1-mapping in children with subclinical anthracycline cardiotoxicity: relationship to exercise capacity, cumulative dose and remodeling. J Cardiovasc Magn Reson. 2013;15:48.

47. Ylanen K, Poutanen T, Savikurki-Heikkila P, Rinta-Kiikka I, Eerola A, Vettenranta K. Cardiac magnetic resonance imaging in the evaluation of the late effects of anthracyclines among long-term survivors of childhood cancer. J Am Coll Cardiol. 2013;61(14):1539-47.

48. van der Meer RW, Lamb HJ, Smit JW, de Roos A. MR imaging evaluation of cardiovascular risk in metabolic syndrome. Radiology. 2012;264(1):21-37.

49. Cavalcante JL, Lima JA, Redheuil A, Al-Mallah MH. Aortic stiffness: current understanding and future directions. J Am Coll Cardiol. 2011;57(14):1511-22. 